\title{
Carbon Dioxide: A Pre-Requisite for Increased Hepatic Glucose Production in Type 2 Diabetes
}

\author{
Ibrahim SI* \\ Department of Biochemistry, College of Medicine and Health Sciences, Federal \\ University, Nigeria \\ *Corresponding author: Ibrahim Salihu Ismail, Department of Biochemistry, \\ College of Medicine and Health Sciences, Federal University, Dutse, Nigeria, Tel: \\ +2348036316108; Email: salihuringim@yahoo.com
}

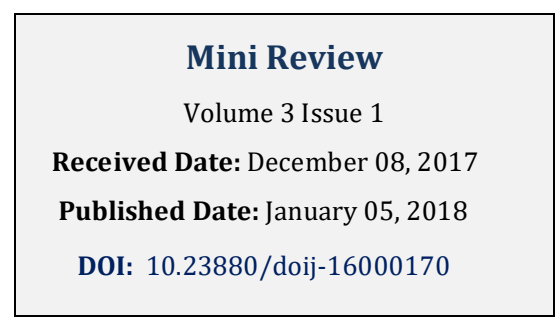

\begin{abstract}
Type 2 diabetic patient have an increased rate of production of hepatic glucose which leads to persistent hyperglycemia. The increase in hepatic glucose production was found to be due to availability and uptake of gluconeogenic substrates, but that alone cannot drive glucose synthesis. Carbon dioxide produced at the end of metabolic combustion of fuel is then used as a substrate for the first step in hepatic glucose production i.e the pyruvate carboxylase reaction.

Decrease in carbon dioxide is associated with decrease in hepatic glucose production. Furthermore, inhibition of carbonic anhydrase which interconverts carbon dioxide and bicarbonate has been found to decrease hepatic glucose synthesis. Therefore, it can be concluded that increased substrate delivery to the liver and increased efficiency of hepatic substrate uptake alone cannot drive glucose synthesis that continues supply of carbon dioxide is necessary for hepatic glucose production.
\end{abstract}

Keywords: Gluconeogenesis; Type 2 Diabetes; Carbon Dioxide; Carbonic Anhydrase

\section{Introduction}

Despite many decades of research in type 2 diabetes mellitus, the disease still remains a serious cause of morbidity and mortality, causing personal suffering and huge economic cost that drives families into poverty. Patients with type 2 diabetes mellitus experience persistent hyperglycemia, which arose from impaired insulin secretion and/or action and also from increase hepatic glucose production. Several antidiabetic drugs act by stimulating insulin secretion and sensitivity whereas some like the biguanide metformin in addition to other functions act by reducing hepatic glucose production. Several studies have demonstrated that increase hepatic glucose production is a major contributor to hyperglycemia in type 2 diabetes.

It has been reported that, fasting hyperglycemia in type 2 diabetes is attributed to excess hepatic glucose output [1-3]. Previous studies indicated that gluconeogenesis is increased in type 2 diabetes $[4,5]$ and that it may account for most of the excess hepatic glucose output [5]. The actual mechanism responsible for increased hepatic glucose production in type 2 diabetes remains to be fully 


\section{Diabetes and Obesity International Journal}

established. Hepatic glucose is produced through a process called gluconeogenesis which mainly uses lactate and alanine as its substrates. Lactate and alanine have been reported to be the major gluconeogenic substrates in man [6,7]. Therefore the rate of hepatic glucose production may solely depend on higher delivery of these substrates to the liver, greater efficiency of hepatic uptake, and increased conversion of these substrates into glucose, or a combination of both processes. Our recent understanding that the enzyme pyruvate carboxylase that catalyses the first reaction of gluconeogenesis requires carbon dioxide as a substrate, as reported by Chegwidden, et al. [8] this indicates that synthesis of glucose from lactate or alanine cannot proceed no matter the amount of the substrate without the availability of carbon dioxide. This so because the lactate that has been produced from the muscle and other glycolytic cells has to be converted to pyruvate first before it can be carboxylated to oxaloacetate by pyruvate carboxylase which requires carbon dioxide as a substrate, before it can then proceed to the next reaction of phosphoenol pyruvate carboxykinase to generate phosphoenol pyruvate which subsequently leads to glucose generation.

There is no doubt that one of the defining features of type 2 diabetes is impaired insulin secretion or action which manifest itself as hyperglycemia due to failure of insulin to get glucose across the cell in order to generate energy and other intermediates that are required in other biosynthetic pathways, one of such intermediates is carbon dioxide.

Carbon dioxide is a molecule that is excreted as a byproduct of glucose oxidation in the mitochondria. Because of its toxic nature at high concentration carbon dioxide cannot be allowed to accumulate, it is therefore transported via the red blood cells and expired into the air through the lungs. Accumulation of carbon dioxide leads to a condition known as hypercapnia, which is characterized by a decrease in blood $\mathrm{pH}$. The interconversion of $\mathrm{CO}_{2}$ and $\mathrm{HCO}_{3}$-is catalyzed by carbonic anhydrase, a ubiquitous enzyme that plays a very important role in providing bicarbonateas substrate for carboxylation in different essential metabolic pathways which include gluconeogenesis and synthesis of some amino acids (pyruvate carboxylase) lipogenesis (pyruvate carboxylase and acetyl coA carboxylase), ureagenesis (carbamoyl synthase I) and pyrimidine synthesis (Carbamoyl phosphate synthase II) [8].

Pyruvate carboxylase mediates the first reaction in gluconeogenesis from pyruvate. It has been shown that pyruvate carboxylase activity was blocked by ethoxzolamide, a carbonic anhydrase inhibitor, in mitochondria isolated from liver from starved guinea pigs [9]. Earlier studies had suggested that sulfonamides another class of carbonic anhydrase inhibitors, inhibit pyruvatecarboxylase activity directly [10]. These studies suggested that the effect of the carbonic anhydrase (CA) inhibitor ethoxzolamide is on mitochondrial $\mathrm{CA}$, not pyruvate carboxylase. These suggest that carbonic anhydrase inhibitor does not have any effect on pyruvate carboxylase, but the carbonic anhydrase itself. Therefore, it's not the inhibition of carbonic anhydrase that blocks gluconeogenesis but lack of provision of bicarbonate which is required by pyruvate carboxylase for gluconeogenesis to proceed. Dodgson and Forester [11] also demonstrated that glucose production in hepatocytes was blocked by carbonic anhydrase inhibitor ethoxzolamide, further indicating that hepatic glucose synthesis only proceeds when $\mathrm{HCO}_{3}-\mathrm{CO}_{2}$ is made available by carbonic anhydrase. This shows that availability and efficient hepatic lactate uptake alone cannot drive hepatic glucose production. Thus, de novo synthesis of $\mathrm{HCO}_{3}{ }^{-}$within the mitochondrial compartment is required for providing substrate for pyruvate carboxylase in the gluconeogenic pathway in the liver $[9,11]$. Even though mitochondrial diseases are often associated with defects in the oxidative phosphorylation [12], the above studies suggest the possibility that the mitochondrial CAs could serve as targets for modulating gluconeogenesis, mainly by regulating the amount of carbon dioxide supplied for pyruvate carboxylase. Mitochondrial dysfunction has been observed in the liver, muscle, adipose tissue and even the brain, including and the hypothalamus, in rodents and humans with obesity, T2DM and metabolic syndrome [13].

A recent study conducted by Carmen, et al. [14], found that defective hepatic bicarbonate production leads to hypoglycemia, elevated lactate level, metabolic acidosis and hyperammonemia, further confirming bicarbonate as a necessary requirement for hepatic glucose synthesis. Thus carbonic anhydrase must keep the $\left[\mathrm{HCO}_{3}^{-}\right] /\left[\mathrm{CO}_{2}\right]$ ratio constant. $\mathrm{CO}_{2}$ and $\mathrm{HCO}_{3}{ }^{-}$, which are continuously generated during the oxidation of energy fuels, need to be eliminated from the body at the same velocity as they are generated metabolically. Whereas the complete oxidation of carbohydrates yields $\mathrm{CO}_{2}$ and water as the only products and the lungs fulfill the role of regulating $\mathrm{PCO}_{2}$, to prevent accumulation through respiratory regulation. Adequate excretion of $\mathrm{CO}_{2}$ is fundamental to normal body function, disruption of any part of $\mathrm{CO}_{2}$ excretion process whether directly or indirectly can result in metabolic acidosis.

As early as $1966 \mathrm{Hems}$, et al. [15] showed that gluconeogenesis from lactate in the isolated perfused rat 


\section{Diabetes and Obesity International Journal}

liver was markedly inhibited when the $\mathrm{pH}$ of the perfusate was less than 7.1 (i.e slightly acidic). In 1977, Iles et al. [16], studied gluconeogenesis from lactate or pyruvate in perfused livers from starved rats at perfusate $\mathrm{pH} 7.4$ and under conditions simulating uncompensated metabolic acidosis (perfusate pH 6.7-6.8). They showed that in 'acidotic' perfusions gluconeogenesis from lactate or pyruvate were decreased and suggested that the effect of acidosis was exerted at a stage preceding phosphoenolpyruvate. It had already been shown that hepatic phosphoenolpyruvate carboxykinase activity has been unchanged in animals rendered acidotic by administration of $\mathrm{NH}_{4} \mathrm{Cl}$ [17-19].

The finding of Iles et al. that glucose output was decreased by $51.3 \%$, intracellular oxaloacetate concentration was lowered by $39 \%$ and suggested that total intracellular oxaloacetate concentration was significantly decreased in the acidotic livers perfused with lactate. And also the findings of Scrutton \& Soling [20,21] that the activity of pyruvate carboxylase is markedly decreased by a fall in $\mathrm{pH}$ from 7.8 to 6.9 points toward the indispensable requirement of bicarbonate as a modulator of hepatic glucose production. The golden question is what is the connection between acidosis, bicarbonate concentration and reduction in hepatic glucose production?

Change in $\mathrm{pH}$ from a normal value of 7.4 to 7.2 results in a $60 \%$ increase in hydrogen ion concentration $\left(\mathrm{H}^{+}\right)$ from $40-63 \mathrm{nmol} / \mathrm{l}$ and a further increase to $100 \mathrm{nmol} / \mathrm{l}$ at a $\mathrm{pH}$ of 7.0 [22]. Thus, clearance of $\left(\mathrm{H}^{+}\right)$becomes necessary in order to restore internal homeostasis which is fundamental for maintaining life. Hydrogen ion concentration is particularly tightly regulated both intracellularly and extracellularly by buffers. One of such buffers is bicarbonate that regulates acidosis at the metabolic level. This same bicarbonate is the one required by pyruvate carboxylate for gluconeogenesis. Reduction in hepatic glucose production in acidosis may be due low bicarbonate concentration that is absolutely required to titrate the high hydrogen concentration in the body at the metabolic level or by loss of bicarbonate rich fluid through the urine. This reduction in bicarbonate will deprive the enzyme pyruvate carboxylase of its substrate for continued hepatic glucose production and thus result in reduced rate of gluconeogenesis. This shows that despite availability of gluconeogenic substrate such as lactate and alanine, gluconeogenesis will not proceed unless bicarbonate is sufficiently available to drive the synthesis of glucose.

\section{Conclusion}

The increase in hepatic glucose production in type 2 diabetes is dependent upon the continued supply of carbon dioxide, despite the availability of gluconeogenic substrates. It is clear that carbon dioxide is the modulator of hepatic glucose production. Therefore it is important to consider the role played by carbon dioxide when designing new antidiabetic drugs that reduces hepatic glucose production.

\section{Search Criteria}

Search strategy: We searched google (1960-2017). We used the search terms 'diabetes' and 'carbonic anhydrase' or 'bicarbonate' or 'acidosis' or incombination with the terms 'hepatic glucose production' or 'gluconeogenesis'.

\section{Conflict of Interest}

The authors declare no conflict of interest.

\section{References}

1. Best JD, Judzewitsch RG, Pfeifer MA, Beard JC, Halter JB, et al. (1982) The effect of chronic sulfonylurea therapy on hepatic glucose production in noninsulindependent diabetes. Diabetes 31(4): 333-338.

2. DeFronzo RA, Simonson D, Ferrannini E (1982) Hepatic and peripheral insulin resistance: a common feature of type II (noninsulin-dependent) and Type I (insulin-dependent) diabetes mellitus. Diabetologia 23(4): 313-319.

3. Campbell PJ, Mandarino LJ, Gerich JE (1988) Quantification of the relative impairment in actions of insulin on hepatic glucose production and peripheral glucose uptake in noninsulin-dependent diabetes mellitus. Metab Clin Exp 37(1): 15-22.

4. Zawadzki JK, Wolfe RR, Mott DM, Lillioja S, Howard $\mathrm{BV}$, et al. (1988) Increased rate of Cori cycle in obese subjects with NIDDM and effects of weight reduction. Diabetes 37(2): 154-159.

5. Consoli A, Nurjhan N, Capani F, Gerich J (1989) Predominant Predominant role ofgluconeogenesis in increased hepatic glucose production in NIDDM. Diabetes 38(5): 550-557.

6. Kreisberg RA (1972) Glucose-lactate inter-relations in man. N Engl J Med 287(3): 132-137. 


\section{Diabetes and Obesity International Journal}

7. Gerich J, Haymond M, Rizza R, Verdonk C, Miles J (1981) Hormonal and substrate determinants of hepatic glucose production in man. In: Veneziale C (Ed.), The Regulation of Carbohydrate Formation and Utilization in Mammals. University Park Press, Baltimore, pp: 419-457.

8. Chegwidden WR, Dodgson SJ, Spencer IM (2000) The roles of carbonic anhydrase in metabolism, cell growth and cancer in animals. EXS 90: 343-363.

9. Dodgson SJ, Forster RE 2nd (1986) Inhibition of CA V decreases glucose synthesis from pyruvate. Arch Biochem Biophys 251(1): 198-204.

10. Cao TP, Rous S (1978) Action of acetazolamide on liver pyruvate carboxylase activity, glycogenolysis and gluconeogenesis of mice. Int J Biochem 9(8): 603605.

11. Dodgson SJ, Forster RE 2nd (1985) Carbonic anhydrase: inhibition results in decreased urea production by hepatocytes. J Appl Physiol 60(2): 646652.

12. Wallace DC (1999) Mitochondrial diseases in man and mouse. Science 283: 1482-1488.

13. Patti ME, Corvera S (2010) The role of mitochondria in the pathogenesis of type 2 diabetes. Endocr Rev 31(3): 364-395.

14. Diez-Fernandez C, Rüfenacht V, Santra S, Lund AM, Santer R, et al. (2016) Defective hepatic bicarbonate production due to carbonic anhydraseVA deficiency leads to early-onset life-threatening metabolic crisis. Genet Med 18(10): 991-1000.
15. Hems R, Ross BD, Berry MN, Krebs HA (1966) Gluconeogenesis in the perfused rat liver. Biochem J 101(2): 284-292.

16. Iles RA, Cohen RD, Rist AH, Baron PG (1977) The Mechanism of Inhibition by Acidosis of Gluconeogenesis from Lactate in Rat Liver. Biochem J 164(1): 185-191.

17. Alleyne GA, Scullard GH (1969) Renal metabolic response to acid base changes. I. Enzymatic control of ammoniagenesis in the rat. J Clin Invest 48(2): 364370.

18. Kamm DE, Cahill GF (1969) Effect of acid-base status on renal and hepatic gluconeogenesis in diabetes and fasting. Am J Physiol 216(5): 1207-1212.

19. Longshaw ID, Alleyne GA, Pogson CI (1972) The effect of steroids and ammonium chloride acidosis on phosphoenolpyruvate carboxykinase in rat kidney cortex. II. The kinetics of enzyme induction. J Clin Invest 51(9): 2284-2291.

20. Scrutton MC, Utter MF (1967) Pyruvate carboxylase. IX. Some properties of the activation by certain acyl derivatives of coenzyme A. J Biol Chem 242(8): 17231735.

21. Soling HD, Willms B, Kleineke J, Gehlhoff M (1970) Regulation of Gluconeogenesis in the Guinea Pig Liver. Eur J Biochem 16(2): 289-302.

22. Boyd JH, Walley KR (2008) Is there a role for sodium bicarbonate in treating lactic acidosisfrom shock? Curr Opin Crit Care 14(4): 379-383. 